\title{
NUMERICAL MODELLING OF A DYNAMIC CONTACT PROBLEM WITH NORMAL DAMPED RESPONSE AND UNILATERAL CONSTRAINT
}

\author{
MikaËL BARBoteu \\ Laboratoire de Mathématiques et Physique, University of Perpignan Via Domitia, Perpignan, France \\ e-mail: barboteu@univ-perp.fr \\ YOUSSEF OUAFIK \\ Université Cadi Ayyad, ENSA Safi, Maroc \\ e-mail:y.ouafik@uca.ma \\ Mircea Sofonea \\ Laboratoire de Mathématiques et Physique, University of Perpignan Via Domitia, Perpignan, France \\ e-mail: sofonea@univ-perp.fr
}

\begin{abstract}
We consider a mathematical model which describes the dynamic evolution of a viscoelastic body in frictional contact with an obstacle. The contact is modelled with normal damped response and unilateral constraint for the velocity field, associated to a version of Coulomb's law of dry friction. Our aim is to present a detailed description of the numerical modelling of the problem. To this end, we use a penalty method to approximate the constraint. Then, we provide numerical simulations in the study of a two-dimensional example and compare the penalty model with the original one.
\end{abstract}

Keywords: dynamic contact, viscoelastic material, frictional contact, normal damped response, unilateral constraint, penalty method, numerical simulations

\section{Introduction}

Contact problems involving deformable bodies arise in many industrial processes as well as in everyday life. For this reason, they have been widely studied in the recent years, with various constitutive laws and boundary conditions, including the normal compliance condition. The studies concern both the mechanical, the mathematical and numerical modelling of the corresponding boundary value problems. References in the field include Oden and Martins (1985), Han et al. (2001, 2016), Han and Sofonea (2002), Laursen (2002), Hlaváček et al. (1988), Barboteu et al. (2015, 2016a,b), Barboteu and Danan (2016), among others. The so-called normal damped response condition represents a version of the normal compliance condition, expressed in terms of velocity. Such a condition seems to be appropriate when contact surfaces are lubricated, as mentioned in (Barboteu and Danan, 2016; Barboteu et al., 2016b; Han et al., 2016; Shillor et al., 2004).

In this current paper, we consider a mathematical model which describes dynamic frictional contact between a body and a deformable foundation. We describe the material behavior with the Kelvin-Voigt viscoelastic constitutive law. The frictional contact is modelled with a normal damped response condition with unilateral constraint for the velocity field associated to a version of Coulomb's law of dry friction. These non standard contact conditions could model the contact with the deformable foundation covered by a lubricant, say oil, as already mentionned.

The current work represents a continuation of (Barboteu et al., 2015, 2016a,b). Its aim is to provide the numerical modelling of the dynamic frictional contact problem supported by numerical simulations. We present a ful discretization of the problem and we describe details 
of the numerical algorithm we use. The main novelty of our work arises from the fact that we study two problems: an original problem constructed by considering a combination of the normal damped response law with unilateral constraint condition in velocity, and a penalty one constructed with the normal damped response law without a constraint. In this paper, we provide a reliable comparison between numerical solutions of the approximate frictional contact problem and the original one. Finally, we present numerical simulations which validate our approximation method and give information on the mechanical behaviour of the solution.

The paper is organized as follows. In Section 2, we present the original model and provide its variational formulation. Then we introduce the penalty problem and its variational formulation. In Section 3, we introduce a hybrid variational formulation of the two above mentioned problems. A fully discrete scheme is presented in Section 4, based on the finite element method to approximate the spatial variable and the Euler scheme to discretize the time derivatives. Finally, in Section 5, we present numerical simulations in the study of a two-dimensional test problem.

\section{The model and its penalty version}

We start by presenting the notation and the preliminary material we need in the rest of the paper. Denote by $\mathbb{S}^{d}$ the space of second order symmetric tensors on $\mathbb{R}^{d}$ and by "." and $\|\cdot\|$ the inner product and the Euclidean norms on the spaces $\mathbb{R}^{d}$ and $\mathbb{S}^{d}$, respectively. Let $\Omega \subset \mathbb{R}^{d}$, $d=1,2,3$ be the domain occupied by a viscoelastic body in the reference configuration, with a smooth boundary $\Gamma=\partial \Omega$. We denote by $\boldsymbol{\nu}$ the unit outer normal vector to $\Gamma$ and assume that $\Gamma$ is decomposed into three measurable parts $\Gamma_{1}, \Gamma_{2}, \Gamma_{3}$, such that meas $\left(\Gamma_{1}\right)>0$. Let $[0, T]$ be the time interval of interest, with $T>0$. We denote by $\mathbf{x} \in \Omega$ and $t \in[0, T]$ the spatial and the time variable, respectively, and, for simplicity, we do not indicate the dependence of the functions on $\mathbf{x}$ and $t$. Moreover, a dot above a variable will represent the derivative with respect to time. Finally, we denote by $\mathbf{u}$ the displacement field, by $\boldsymbol{\sigma}$ the stress tensor, and $\varepsilon(\mathbf{u})=\left(\varepsilon_{i j}(\mathbf{u})\right)_{i, j=1}^{d}-$ the linearized strain field, i.e.

$$
\varepsilon_{i j}(\mathbf{u})=\frac{1}{2}\left(\frac{\partial u_{i}}{\partial x_{j}}+\frac{\partial u_{j}}{\partial x_{i}}\right)
$$

The body is assumed to be viscoelastic and, therefore, we use the constitutive law

$$
\boldsymbol{\sigma}=\mathcal{A} \varepsilon(\dot{\mathbf{u}})+\mathcal{B} \varepsilon(\mathbf{u}) \quad \text { in } \quad \Omega \times(0, T)
$$

already used in (Duvaut and Lions, 1976), for instance. Here $\mathcal{A}$ and $\mathcal{B}$ are the fourth-order viscosity and elastic tensors, respectively. Since the process is dynamic, the balance equation of the stress field is given by

$$
\operatorname{Div} \boldsymbol{\sigma}+\mathbf{f}_{0}=\rho \ddot{\mathbf{u}} \quad \text { in } \quad \Omega \times(0, T)
$$

Here, $\mathbf{f}_{0}$ is density of the body forces and $\rho$ stands for density of the material, assumed to be constant for simplicity. Moreover, Div represent the divergence operator, i.e. $\operatorname{Div}(\boldsymbol{\sigma})=\left(\sigma_{i j, j}\right)$.

On $\Gamma_{1}$, the body is clamped and, therefore,

$$
\mathbf{u}=\mathbf{0} \quad \text { on } \quad \Gamma_{1} \times(0, T)
$$

Moreover, we assume that a surface force of density $\mathbf{f}_{2}$ acts on $\Gamma_{2}$, i.e.

$$
\boldsymbol{\sigma} \boldsymbol{\nu}=\mathbf{f}_{2} \quad \text { on } \quad \Gamma_{2} \times(0, T)
$$


The part $\Gamma_{3}$ of the boundary represents the potential contact surface and is assumed to be given. There, the body can arrive in contact with a piston or a device, the so-called foundation. Considering the case of an evolutive (say growing) contact surface leads to various mathematical difficulties and, therefore, is left open. The boundary conditions on $\Gamma_{3}$ are derived from the following assumptions.

- The obstacle prevents motion of the body in such a way that the normal velocity is restricted by an unilateral constraint, i.e.

$$
\dot{u}_{\nu} \leqslant g
$$

where $\dot{u}_{\nu}=\dot{\mathbf{u}} \cdot \boldsymbol{\nu}$ denotes the normal component of $\dot{\mathbf{u}}$ on $\Gamma$ and $g>0$ represents a given bound. Here, we assume the non homogeneous case and, therefore, $g$ is a function which could depend on the spatial variable $\mathbf{x} \in \Gamma_{3}$.

- When the body moves in the opposite direction of the obstacle then the reaction of the obstacle vanishes. Therefore,

$$
\dot{u}_{\nu}<0 \Longrightarrow \sigma_{\nu}=0 \quad \boldsymbol{\sigma}_{\tau}=\mathbf{0}
$$

where $\sigma_{\nu}$ and $\sigma_{\tau}$ denote the normal and the tangential components of the stress on $\Gamma$, i.e. $\sigma_{\nu}=(\boldsymbol{\sigma} \boldsymbol{\nu}) \cdot \boldsymbol{\nu}$ and $\boldsymbol{\sigma}_{\tau}=\boldsymbol{\sigma} \boldsymbol{\nu}-\sigma_{\nu} \boldsymbol{\nu}$.

- When the body moves towards the obstacle, the contact is described with the normal damped response condition associated to Coulomb's law of dry friction as far as the normal velocity does not reach the bound $g$. Therefore,

$$
\begin{aligned}
& 0 \leqslant \dot{u}_{\nu}<g \Longrightarrow-\sigma_{\nu}=p\left(\dot{u}_{\nu}\right) \quad\left\|\boldsymbol{\sigma}_{\tau}\right\| \leqslant \mu\left|\sigma_{\nu}\right| \\
& -\boldsymbol{\sigma}_{\tau}=\mu\left|\sigma_{\nu}\right| \frac{\dot{\mathbf{u}}_{\tau}}{\left\|\dot{\mathbf{u}}_{\tau}\right\|} \quad \text { if } \quad \dot{\mathbf{u}}_{\tau} \neq \mathbf{0}
\end{aligned}
$$

Here, $p$ represents a positive function such that $p(r)=0$ for $r \leqslant 0$ and $\mu$ denotes the coefficient of friction. Details on the normal damped response contact condition associated to Coulomb's law of dry friction can be found in (Han and Sofonea, 2002; Shillor et al., 2004; Sofonea and Matei, 2012), for instance.

- When the normal velocity reaches the bound $g$, then the normal stress is larger than $p(g)$ and, moreover, friction follows the Tresca law with the friction bound $F_{b}$. Therefore,

$$
\begin{aligned}
& \dot{u}_{\nu}=g \Longrightarrow-\sigma_{\nu} \geqslant p(g) \quad\left\|\boldsymbol{\sigma}_{\tau}\right\| \leqslant F_{b} \\
& -\boldsymbol{\sigma}_{\tau}=F_{b} \frac{\dot{\mathbf{u}}_{\tau}}{\left\|\dot{\mathbf{u}}_{\tau}\right\|} \quad \text { if } \quad \dot{\mathbf{u}}_{\tau} \neq \mathbf{0}
\end{aligned}
$$

— To accommodate conditions (2.7) and (2.8), we assume the compatibility condition

$$
F_{b}=\mu p(g)
$$

which guarantees continuity of the friction bound. Note that conditions (2.7) and (2.8) show a natural transition from the Coulomb law (which is valid as far as $0 \leqslant \dot{u}_{\nu}<g$ ) to the Tresca friction law (which is valid when $\dot{u}_{\nu}=g$ ).

A careful examination of conditions (2.5)-(2.9) reveals that they can be written, equivalently, as follows

$$
\begin{array}{lcc}
\dot{u}_{\nu} \leqslant g & \sigma_{\nu}+p\left(\dot{u}_{\nu}\right) \leqslant 0 \quad\left(\dot{u}_{\nu}-g\right)\left(\sigma_{\nu}+p\left(\dot{u}_{\nu}\right)\right)=0 \\
\left\|\boldsymbol{\sigma}_{\tau}\right\| \leqslant \mu\left|\sigma_{\nu}\right| \quad & -\boldsymbol{\sigma}_{\tau}=\mu\left|\sigma_{\nu}\right| \frac{\dot{\mathbf{u}}_{\tau}}{\left\|\dot{\mathbf{u}}_{\tau}\right\|} \quad \text { if } \quad \dot{\mathbf{u}}_{\tau} \neq \mathbf{0}
\end{array}
$$


Moreover, conditions (2.10) are equivalent to

$$
\begin{aligned}
& -\sigma_{\nu}(t) \in p\left(\dot{u}_{\nu}(t)\right)+\partial I_{(-\infty, g]}\left(\dot{u}_{\nu}(t)\right) \quad \text { on } \quad \Gamma_{3} \times(0, T) \\
& -\boldsymbol{\sigma}_{\tau}(t) \in \mu p\left(\dot{u}_{\nu}(t)\right) \partial\left\|\dot{\mathbf{u}}_{\tau}(t)\right\| \quad \text { on } \quad \Gamma_{3} \times(0, T)
\end{aligned}
$$

respectively. Here $\partial$ represents the subdifferential operator in the sense of convex analysis and $I_{A}$ denotes the indicator function of the set $A \subset \mathbb{R}$. Subdifferential inclusions (2.11) will be considered for the numerical modelling we introduce in Section 3.

Finally, we prescribe the initial displacement and the initial velocity, i.e.

$$
\mathbf{u}(0)=\mathbf{u}_{0} \quad \dot{\mathbf{u}}(0)=\mathbf{u}_{1} \quad \text { in } \quad \Omega
$$

where $\mathbf{u}_{0}$ and $\mathbf{u}_{1}$ are given functions defined on $\Omega$.

We now gather relations (2.1)-(2.4), (2.10) and (2.12) to obtain the following formulation of the dynamic frictional contact problem we consider in this paper.

Problem P. Find a displacement field $\mathbf{u}: \Omega \times(0, T) \rightarrow \mathbb{R}^{d}$ and a stress field $\boldsymbol{\sigma}: \Omega \times(0, T) \rightarrow \mathbb{S}^{d}$ such that

$$
\begin{aligned}
& \boldsymbol{\sigma}=\mathcal{A} \varepsilon(\dot{\mathbf{u}})+\mathcal{B} \varepsilon(\mathbf{u}) \quad \text { in } \quad \Omega \times(0, T) \\
& \operatorname{Div} \boldsymbol{\sigma}+\mathbf{f}_{0}=\rho \ddot{\mathbf{u}} \quad \text { in } \quad \Omega \times(0, T) \\
& \mathbf{u}=\mathbf{0} \quad \text { on } \quad \Gamma_{1} \times(0, T) \\
& \boldsymbol{\sigma} \boldsymbol{\nu}=\mathbf{f}_{2} \quad \text { on } \quad \Gamma_{2} \times(0, T) \\
& \dot{u}_{\nu} \leqslant g \quad \sigma_{\nu}+p\left(\dot{u}_{\nu}\right) \leqslant 0 \quad\left(\dot{u}_{\nu}-g\right)\left(\sigma_{\nu}+p\left(\dot{u}_{\nu}\right)\right)=0 \quad \text { on } \quad \Gamma_{3} \times(0, T) \\
& \left\|\boldsymbol{\sigma}_{\tau}\right\| \leqslant \mu p\left(\dot{u}_{\nu}\right) \quad-\boldsymbol{\sigma}_{\tau}=\mu p\left(\dot{u}_{\nu}\right) \frac{\dot{\mathbf{u}}_{\tau}}{\left\|\dot{\mathbf{u}}_{\tau}\right\|} \quad \text { if } \quad \dot{\mathbf{u}}_{\tau} \neq \mathbf{0} \quad \text { on } \quad \Gamma_{3} \times(0, T) \\
& \mathbf{u}(0)=\mathbf{u}_{0} \quad \dot{\mathbf{u}}(0)=\mathbf{u}_{1} \quad \text { in } \quad \Omega
\end{aligned}
$$

We now turn to the variational formulation of Problem $P$ which is the starting point for the numerical modelling based on the finite element discretization. To this end, we use the notaion $H=\left[L^{2}(\Omega)\right]^{d}$ and we introduce the spaces

$$
\begin{aligned}
& V=\left\{\mathbf{v} \in\left[H^{1}(\Omega)\right]^{d} ; \mathbf{v}=\mathbf{0} \quad \text { on } \quad \Gamma_{1}\right\} \\
& Q=\left\{\boldsymbol{\tau}=\left(\tau_{i j}\right)_{i, j=1}^{d} \in\left[L^{2}(\Omega)\right]^{d \times d} ; \quad \tau_{i j}=\tau_{j i}, \quad i, j=1, \ldots, d\right\}
\end{aligned}
$$

The spaces $H, V$ and $Q$ are real Hilbert spaces endowed with the canonical inner products given by

$$
(\boldsymbol{\theta}, \boldsymbol{\eta})_{H}=\int_{\Omega} \boldsymbol{\theta} \cdot \boldsymbol{\eta} d x \quad(\mathbf{u}, \mathbf{v})_{V}=\int_{\Omega} \boldsymbol{\varepsilon}(\mathbf{u}) \cdot \boldsymbol{\varepsilon}(\mathbf{v}) d x \quad(\boldsymbol{\sigma}, \boldsymbol{\tau})_{Q}=\int_{\Omega} \boldsymbol{\sigma} \cdot \boldsymbol{\tau} d x
$$

On the density of volume forces and surface tractions, we assume that

$$
\mathbf{f}_{0} \in C([0, T] ; H) \quad \mathbf{f}_{2} \in C\left([0, T] ;\left[L^{2}\left(\Gamma_{2}\right)\right]^{d}\right)
$$

and, using the Riesz representation theorem, we define the linear function $\mathbf{f}:[0, T] \rightarrow V$ by the equality

$$
(\mathbf{f}(t), \mathbf{w})_{V}=\int_{\Omega} \mathbf{f}_{0}(t) \cdot \mathbf{w} d \mathbf{x}+\int_{\Gamma_{2}} \mathbf{f}_{2}(t) \cdot \mathbf{w} d \Gamma \quad \forall \mathbf{w} \in V
$$


Note that assumption (2.20) implies that $\mathbf{f} \in C([0, T] ; V)$. Here and below notation $C([0, T] ; X)$ represents the space of continuous functions defined on $[0, T]$ with values to $X$.

Next, we denote by $j: U \times U \rightarrow \mathbb{R}$ the function given by

$$
j(\mathbf{u}, \mathbf{w})=\int_{\Gamma_{3}} \mu p\left(u_{\nu}\right)\left\|\mathbf{w}_{\tau}\right\| d \Gamma \quad \forall \mathbf{u}, \mathbf{w} \in U
$$

where, $U:=\left\{\mathbf{w} \in V: w_{\nu} \leqslant g\right.$ a.e. on $\left.\Gamma_{3}\right\}$ and $w_{\nu}=\mathbf{w} \cdot \boldsymbol{\nu}$ and $\mathbf{w}_{\tau}=\mathbf{w}-w_{\nu} \boldsymbol{\nu}$, for all $\mathbf{w} \in V$.

Now, using standard arguments based on the Green formula, we obtain the following variational formulation of Problem $P$.

Problem $P_{V}$. Find a displacement field $\mathbf{u}:[0, T] \rightarrow V$ such that $\dot{\mathbf{u}}(t) \in U$ for all $t \in[0, T]$, $\mathbf{u}(0)=\mathbf{u}_{0}, \dot{\mathbf{u}}(0)=\mathbf{u}_{1}$ and the inequality below holds, for all $t \in(0, T)$ :

$$
\begin{aligned}
& (\rho \ddot{\mathbf{u}}(t), \mathbf{w}-\dot{\mathbf{u}}(t))_{H}+(\mathcal{A} \varepsilon(\dot{\mathbf{u}}(t)), \varepsilon(\mathbf{w})-\varepsilon(\dot{\mathbf{u}}(t)))_{Q} \\
& \quad+(\mathcal{B} \varepsilon(\mathbf{u}(t)), \boldsymbol{\varepsilon}(\mathbf{w})-\varepsilon(\dot{\mathbf{u}}(t)))_{Q}+\left(p\left(\dot{u}_{\nu}(t)\right), w_{\nu}-\dot{u}_{\nu}(t)\right)_{L^{2}\left(\Gamma_{3}\right)}+j(\dot{\mathbf{u}}(t), \mathbf{w}) \\
& \quad-j(\dot{\mathbf{u}}(t), \dot{\mathbf{u}}(t)) \geqslant(\mathbf{f}(t), \mathbf{w}-\dot{\mathbf{u}}(t))_{V} \quad \forall \mathbf{w} \in U
\end{aligned}
$$

Our goal in what follows is to provide a penalty method in order to remove the unilateral constraint $\dot{u}_{\nu} \leqslant g$ in (2.17). The penalty form of the Problem $P$ leads to a simpler numerical model which provides a reliable approximation of the solution of the initial problem. Following arguments similar to those in (Chouly and Hid, 2013; Kikuchi and Song, 1981), the penalty contact problem we consider is the following.

Problem $P_{\varepsilon}$. Find a displacement field $\mathbf{u}_{\varepsilon}: \Omega \times(0, T) \rightarrow \mathbb{R}^{d}$ and a stress field $\boldsymbol{\sigma}_{\varepsilon}: \Omega \times(0, T) \rightarrow$ $\mathbb{S}^{d}$ such that

$$
\begin{aligned}
& \boldsymbol{\sigma}_{\varepsilon}=\mathcal{A} \varepsilon\left(\dot{\mathbf{u}}_{\varepsilon}\right)+\mathcal{B} \varepsilon\left(\mathbf{u}_{\varepsilon}\right) \text { in } \Omega \times(0, T) \\
& \operatorname{Div} \boldsymbol{\sigma}_{\varepsilon}+\mathbf{f}_{0}=\rho \ddot{\mathbf{u}}_{\varepsilon} \text { in } \Omega \times(0, T) \\
& \mathbf{u}_{\varepsilon}=\mathbf{0} \quad \text { on } \quad \Gamma_{1} \times(0, T) \\
& \boldsymbol{\sigma}_{\varepsilon} \boldsymbol{\nu}=\mathbf{f}_{2} \text { on } \Gamma_{2} \times(0, T) \\
& -\sigma_{\varepsilon_{\nu}}=\frac{1}{\varepsilon}\left(\dot{u}_{\varepsilon_{\nu}}-g\right)_{+}+p\left(\dot{u}_{\varepsilon_{\nu}}\right) \quad \text { on } \quad \Gamma_{3} \times(0, T) \\
& \left\|\boldsymbol{\sigma}_{\varepsilon_{\tau}}\right\| \leqslant \mu p\left(\dot{u}_{\varepsilon_{\nu}}\right) \quad-\boldsymbol{\sigma}_{\varepsilon_{\tau}}=\mu p\left(\dot{u}_{\varepsilon_{\nu}}\right) \frac{\dot{\mathbf{u}}_{\varepsilon_{\tau}}}{\left\|\dot{\mathbf{u}}_{\varepsilon_{\tau}}\right\|} \text { if } \dot{\mathbf{u}}_{\varepsilon_{\tau}} \neq \mathbf{0} \quad \text { on } \Gamma_{3} \times(0, T) \\
& \mathbf{u}_{\varepsilon}(0)=\mathbf{u}_{0} \quad \dot{\mathbf{u}}_{\varepsilon}(0)=\mathbf{u}_{1} \quad \text { in } \quad \Omega
\end{aligned}
$$

Here and below, $\varepsilon$ represents the penalty parameter assumed to be very small, while $\dot{u}_{\varepsilon_{\nu}}$ and $\sigma_{\varepsilon_{\nu}}$ represent the normal components of the functions $\dot{\mathbf{u}}_{\varepsilon}$ and $\boldsymbol{\sigma}_{\varepsilon}$, respectively. Moreover, $\boldsymbol{\sigma}_{\varepsilon_{\tau}}$ represents the tangential part of the function $\boldsymbol{\sigma}_{\varepsilon}$. Note that Problem $P_{\varepsilon}$ is constructed by using similar ingredients to those used in Problem $P$. The differences arise in the fact that here we replace contact condition (2.17) with its penalty version (2.28) and, therefore, Problem $P_{\varepsilon}$ represents a contact problem with the normal damped response, without a unilateral constraint.

Next, using the notation in equations (2.21) and (2.22) and a similar argument to that used in the case of Problem $P$, we obtain the following variational formulation of Problem $P_{\varepsilon}$. 
Problem $P_{V}^{\varepsilon}$. Find a displacement field $\mathbf{u}_{\varepsilon}:[0, T] \rightarrow V$ such that $\mathbf{u}_{\varepsilon}(0)=\mathbf{u}_{0}, \dot{\mathbf{u}}_{\varepsilon}(0)=\mathbf{u}_{1}$ and the inequality below holds, for all $t \in(0, T)$ :

$$
\begin{aligned}
& \left(\rho \ddot{\mathbf{u}}_{\varepsilon}(t), \mathbf{w}-\dot{\mathbf{u}}_{\varepsilon}(t)\right)_{H}+\left(\mathcal{A} \varepsilon\left(\dot{\mathbf{u}}_{\varepsilon}(t)\right), \varepsilon(\mathbf{w})-\varepsilon\left(\dot{\mathbf{u}}_{\varepsilon}(t)\right)\right)_{Q} \\
& \quad+\left(\mathcal{B} \varepsilon\left(\mathbf{u}_{\varepsilon}(t)\right), \varepsilon(\mathbf{w})-\varepsilon\left(\dot{\mathbf{u}}_{\varepsilon}(t)\right)\right)_{Q}+\frac{1}{\varepsilon}\left(\left(\dot{u}_{\varepsilon_{\nu}}(t)-g\right), w_{\nu}-\dot{u}_{\varepsilon_{\nu}}(t)\right)_{L^{2}\left(\Gamma_{3}\right)} \\
& \quad+\left(p\left(\dot{u}_{\varepsilon_{\nu}}(t)\right), w_{\nu}-\dot{u}_{\varepsilon_{\nu}}(t)\right)_{L^{2}\left(\Gamma_{3}\right)}+j\left(\dot{\mathbf{u}}_{\varepsilon}(t), \mathbf{w}\right) \\
& \quad-j\left(\dot{\mathbf{u}}_{\varepsilon}(t), \dot{\mathbf{u}}_{\varepsilon}(t)\right) \geqslant\left(\mathbf{f}(t), \mathbf{w}-\dot{\mathbf{u}}_{\varepsilon}(t)\right)_{V} \quad \forall \mathbf{w} \in V
\end{aligned}
$$

Note that Problem $P_{V}$ represents a second order evolutionary quasivariational inequality with unilateral constraints. In contrast, Problem $P_{V}^{\varepsilon}$ represents a second order evolutionary quasivariational inequality without unilateral constraints.

\section{Hybrid variational formulation}

We now turn to a hybrid variational formulation of the model which is more appropriate for the numerical modelling. To this end, consider the trace spaces $X_{\nu}=\left\{v_{\nu_{\Gamma_{3}}}: \mathbf{v} \in V\right\}$ and $X_{\tau}=\left\{\left.\mathbf{v}_{\tau}\right|_{\Gamma_{3}}: \mathbf{v} \in V\right\}$ equipped with their usual norms. Denote by $X_{\nu}^{*}$ and $X_{\tau}^{*}$ the duals of the spaces $X_{\nu}$ and $X_{\tau}$, respectively. Moreover, let $\langle\cdot, \cdot\rangle_{X_{\nu}^{*} \times X_{\nu}}$ and $\langle\cdot, \cdot\rangle_{X_{\tau}^{*} \times X_{\tau}}$ be the corresponding duality pairing mappings.

For the contact conditions, we introduce a function $\varphi_{\nu}: X_{\nu} \rightarrow(-\infty,+\infty]$ and an operator $L: X_{\nu} \rightarrow X_{\nu}^{*}$ defined by

$$
\begin{aligned}
& \varphi_{\nu}\left(u_{\nu}\right)=\int_{\Gamma_{3}} I_{\mathbb{R}_{-}}\left(u_{\nu}-g\right) d a \quad \forall u_{\nu} \in X_{\nu} \\
& \left\langle L u_{\nu}, w_{\nu}\right\rangle_{X_{\nu}^{*} \times X_{\nu}}=\int_{\Gamma_{3}} p\left(u_{\nu}\right) w_{\nu} d a \quad \forall u_{\nu}, \quad w_{\nu} \in X_{\nu}
\end{aligned}
$$

We note that for all $t \in[0, T]$, condition $(2.11)_{1}$ leads to the subdifferential inclusion

$$
-\sigma_{\nu}(t) \in \partial \varphi_{\nu}\left(\dot{u}_{\nu}(t)\right)+L \dot{u}_{\nu}(t) \quad \text { in } \quad X_{\nu}^{*}
$$

where $\partial \varphi$ denotes the subdifferential of $\varphi$.

For the friction law, we introduce a function $\varphi_{\tau}: X_{\tau} \rightarrow(-\infty,+\infty]$ defined by

$$
\varphi_{\tau}\left(\mathbf{u}_{\tau}\right)=\int_{\Gamma_{3}}\left\|\mathbf{u}_{\tau}\right\| d a \quad \forall \mathbf{u}_{\tau} \in X_{\tau}
$$

We also note that for all $t \in[0, T]$, condition $(2.11)_{2}$ leads to the subdifferential inclusion

$$
-\boldsymbol{\sigma}_{\tau}(t) \in \mu p\left(\dot{u}_{\nu}(t)\right) \partial \varphi_{\tau}\left(\dot{\mathbf{u}}_{\tau}(t)\right) \quad \text { in } \quad X_{\tau}^{*}
$$

Inclusions (3.1) and (3.2) suggest introduction of the new unknowns $\lambda_{\nu}$ and $\boldsymbol{\lambda}_{\tau}$, the so-called Lagrange multipliers, related to the contact and friction stresses on the contact surface, respectively. In our formulation, $\lambda_{\nu}$ corresponds to $-\sigma_{\nu}$ and $\boldsymbol{\lambda}_{\tau}$ coresponds to $-\boldsymbol{\sigma}_{\tau}$. Thus, proceeding in a standard way and using inclusions (3.1) and (3.2), we obtain the following hybrid variational formulation of Problem $P$ in terms of three unknown fields. 
Problem $\widetilde{P}_{V}$. Find a displacement field $\mathbf{u}:[0, T] \rightarrow V$ with $\mathbf{u}(0)=\mathbf{u}_{0}, \dot{\mathbf{u}}(0)=\mathbf{u}_{1}$, a normal stress $\lambda_{\nu}:[0, T] \rightarrow X_{\nu}^{*}$ and a tangential stress $\boldsymbol{\lambda}_{\tau}:[0, T] \rightarrow X_{\tau}^{*}$ such that the inequality below holds, for all $t \in(0, T)$ :

$$
\begin{aligned}
& (\rho \ddot{\mathbf{u}}(t), \mathbf{w})_{H}+(\mathcal{A} \varepsilon(\dot{\mathbf{u}}(t)), \varepsilon(\mathbf{w}))_{Q}+(\mathcal{B} \varepsilon(\mathbf{u}(t)), \varepsilon(\mathbf{w}))_{Q} \\
& \quad+\left\langle\lambda_{\nu}(t), w_{\nu}\right\rangle_{X_{\nu}^{*} \times X_{\nu}}+\left\langle\boldsymbol{\lambda}_{\tau}(t), \mathbf{w}_{\tau}\right\rangle_{X_{\tau}^{*} \times X_{\tau}}=(\mathbf{f}(t), \mathbf{w})_{V} \quad \forall \mathbf{w} \in V \\
& -\lambda_{\nu}(t) \in \partial \varphi_{\nu}\left(\dot{u}_{\nu}(t)\right)+L \dot{u}_{\nu}(t) \quad \text { in } X_{\nu}^{*} \\
& -\boldsymbol{\lambda}_{\tau}(t) \in \mu p\left(\dot{u}_{\nu}(t)\right) \partial \varphi_{\tau}\left(\dot{\mathbf{u}}_{\tau}(t)\right) \quad \text { in } \quad X_{\tau}^{*}
\end{aligned}
$$

The hybrid variational formulation of Problem $P_{V}^{\varepsilon}$ can be obtained in a similar way and is as follows.

Problem $\widetilde{P}_{V}^{\varepsilon}$. Find a displacement field $\mathbf{u}_{\varepsilon}:[0, T] \rightarrow V$ with $\mathbf{u}_{\varepsilon}(0)=\mathbf{u}_{0}, \dot{\mathbf{u}}_{\varepsilon}(0)=\mathbf{u}_{1}$ and a tangential stress field $\boldsymbol{\lambda}_{\varepsilon}:[0, T] \rightarrow X_{\tau}^{*}$ such that the inequality below holds, for all $t \in(0, T)$ :

$$
\begin{aligned}
& \left(\rho \ddot{\mathbf{u}}_{\varepsilon}(t), \mathbf{w}\right)_{H}+\left(\mathcal{A} \varepsilon\left(\dot{\mathbf{u}}_{\varepsilon}(t)\right), \varepsilon(\mathbf{w})\right)_{Q}+\left(\mathcal{B} \varepsilon\left(\mathbf{u}_{\varepsilon}(t)\right), \boldsymbol{\varepsilon}(\mathbf{w})\right)_{Q} \\
& \quad+\left\langle\frac{1}{\varepsilon}\left(\dot{\mathbf{u}}_{\varepsilon_{\nu}}(t)-g\right)_{+}+p\left(\dot{u}_{\varepsilon_{\nu}}\right), w_{\nu}\right\rangle_{X_{\nu}^{*} \times X_{\nu}}+\left\langle\boldsymbol{\lambda}_{\varepsilon}(t), \mathbf{w}_{\tau}\right\rangle_{X_{\tau}^{*} \times X_{\tau}}=(\mathbf{f}(t), \mathbf{w})_{V} \quad \forall \mathbf{w} \in V \\
& \boldsymbol{\lambda}_{\varepsilon}(t) \in \mu p\left(\dot{u}_{\varepsilon_{\nu}}(t)\right) \partial \varphi_{\tau}\left(\dot{\mathbf{u}}_{\varepsilon_{\tau}}(t)\right) \quad \text { in } \quad X_{\tau}^{*}
\end{aligned}
$$

Note that Problem $\widetilde{P}_{V}$ is formulated in terms of three unknown fields. In contrast, due to the penalty term, Problem $\widetilde{P}_{V}^{\varepsilon}$ is formulated in terms of two unknown fields.

\section{Numerical approximation and solution algorithm}

\subsection{Numerical approximation}

We now present a fully discrete approximation of Problems $\widetilde{P}_{V}$ and $\widetilde{P}_{V}^{\varepsilon}$. First, in order to approximate the spatial variable, we assume that $\Omega$ is a polygonal domain and we consider a regular triangulation of $\Omega$, denoted by $\mathcal{T}^{h}$, compatible with the boundary decomposition $\Gamma_{1}$, $\Gamma_{2}$ and $\Gamma_{3}$. Here and below, $h>0$ denotes the spatial discretization parameter. Consider the discrete variational space

$$
V^{h}=\left\{\mathbf{v}^{h} \in[C(\bar{\Omega})]^{d} ; \mathbf{v}_{\left.\right|_{T_{t r}}}^{h} \in\left[P_{1}\left(T_{t r}\right)\right]^{d} \quad \forall T_{t r} \in \mathcal{T}^{h}, \mathbf{v}^{h}=\mathbf{0} \text { at the nodes on } \Gamma_{1}\right\}
$$

where $P_{1}(T)$ represents the space of polynomials of the global degree less or equal to one in $T_{t r}$, and let $U^{h}=U \cap V^{h}$. We note that $U^{h}$ can be obtained as

$$
U^{h}=\left\{\mathbf{v}^{h} \in V^{h} ; v_{\nu}^{h} \leqslant g \text { a.e. on } \Gamma_{3}\right\}
$$

The constraint condition $v_{\nu}^{h} \leqslant g$ on the boundary $\Gamma_{3}$ is satisfied at the nodes, i.e. $v_{\nu}^{h} \leqslant g^{I}$, where $g^{I}$ is the linear interpolation of the function $g$. To discretize the time derivatives, we use a uniform partition of $[0, T]$, denoted by $0=t_{0}<t_{1}<\ldots<t_{N}=T$, and let $k$ be the time step size, $k=T / N$. In what follows, we denote $\mathbf{f}_{n}=\mathbf{f}\left(t_{n}\right), \mathbf{u}_{n}=\mathbf{u}\left(t_{n}\right)$. For a sequence $\mathbf{u}^{h k}=\left\{\mathbf{u}_{n}^{h k}\right\}_{n=0}^{N}$, we use the notation $\delta_{n} \mathbf{u}_{n}^{h k}=\left(\mathbf{u}_{n}^{h k}-\mathbf{u}_{n-1}^{h k}\right) / k, n=1, \ldots, N$, for the backward divided differences, as well as the additional notation $\delta \mathbf{u}^{h k}=\left\{\delta_{n} \mathbf{u}_{n}^{h k}\right\}_{n=0}^{N}$. 
We now consider the spaces $X_{\nu}^{h}=\left\{v_{\nu_{\Gamma_{3}}}^{h}: \mathbf{v}^{h} \in V^{h}\right\}$ and $X_{\tau}^{h}=\left\{\mathbf{v}_{\left.\tau\right|_{\Gamma_{3}}}^{h}: \mathbf{v}^{h} \in V^{h}\right\}$ equipped with their usual norm. We also consider the discrete space of piecewise constants $Y_{\nu}^{h} \subset L^{2}\left(\Gamma_{3}\right)$ and $Y_{\tau}^{h} \subset L^{2}\left(\Gamma_{3}\right)^{d}$ related to the discretization of the normal and tangential stress, respectively. Then, conditions (3.1) and (3.2) lead to the following discrete subdifferential inclusions at the time $t_{n}$

$$
\begin{aligned}
& \left.\lambda_{\nu_{n}}^{h k} \in \partial \varphi_{\nu} \delta u_{\nu_{n}}^{h k}\right)+L \delta u_{\nu_{n}}^{h k} \quad \text { in } \quad Y_{\nu}^{h} \\
& \lambda_{\tau_{n}}^{h k} \in \mu p\left(\delta u_{\nu_{n}}^{h k}\right) \partial \varphi_{\tau}\left(\delta \mathbf{u}_{\tau_{n}}^{h k}\right) \quad \text { in } \quad Y_{\tau}^{h}
\end{aligned}
$$

More details about this discretization step can be found in (Khenous et al., 2006).

Let $\mathbf{u}_{0}^{h} \in V^{h}$ and $\mathbf{u}_{1}^{h} \in V^{h}$ be finite element approximations of $\mathbf{u}_{0}$ and $\mathbf{u}_{1}$, respectively. Using the previous notation and the backward Euler finite difference $\delta \mathbf{v}_{n}=\left(\mathbf{v}_{n}-\mathbf{v}_{n-1}\right) / k$, the fully discrete approximation of the Problem $\widetilde{P}_{V}$ at the time $t_{n}$ is the following.

Problem $\widetilde{P}_{V}^{h k}$. Find a velocity field $\mathbf{v}^{h k}=\left\{\mathbf{v}_{n}^{h k}\right\}_{n=0}^{N} \subset V^{h}$, a normal stress $\lambda_{\nu_{n}}^{h k}=\left\{\lambda_{\nu_{n}}^{h k}\right\}_{n=0}^{N}$ $\subset Y_{\nu}^{h}$ and a tangential stress $\boldsymbol{\lambda}_{\tau_{n}}^{h k}=\left\{\boldsymbol{\lambda}_{\tau_{n}}^{h k}\right\}_{n=0}^{N} \subset Y_{\tau}^{h}$ such that, for all $n=1, \ldots, N$

$$
\begin{aligned}
& \left(\frac{\rho}{k}\left(\mathbf{v}_{n}^{h k}-\mathbf{v}_{n-1}^{h k}\right), \mathbf{w}^{h}\right)_{H}+\left(\mathcal{A} \varepsilon\left(\mathbf{v}_{n}^{h k}\right), \varepsilon\left(\mathbf{w}^{h}\right)\right)_{Q}+\left(\mathcal{B} \varepsilon\left(\mathbf{u}_{n}^{h k}\right), \boldsymbol{\varepsilon}\left(\mathbf{w}^{h}\right)\right)_{Q} \\
& \quad+\int_{\Gamma_{3}} \lambda_{\nu_{n}}^{h k} w_{\nu}^{h k} d a+\int_{\Gamma_{3}} \lambda_{\tau_{n}}^{h k} \cdot \mathbf{w}_{\tau}^{h k} d a=\left(\mathbf{f}_{n}^{h k}, \mathbf{w}^{h}\right)_{V} \quad \forall \mathbf{w}^{h} \in V^{h} \\
& \lambda_{\nu_{n}}^{h k} \in \partial \varphi_{\nu}\left(v_{\nu_{n}}^{h k}\right)+L v_{\nu_{n}}^{h k} \quad \text { in } \quad Y_{\nu}^{h} \\
& \lambda_{\tau_{n}}^{h k} \in \mu p\left(v_{\nu_{n}}^{h k}\right) \partial \varphi_{\tau}\left(\mathbf{v}_{\tau_{n}}^{h k}\right) \quad \text { in } \quad Y_{\tau}^{h}
\end{aligned}
$$

Here, $\mathbf{u}_{0}^{h k}=\mathbf{u}_{0}^{h}, \mathbf{v}_{0}^{h k}=\mathbf{u}_{1}^{h}$ and $\mathbf{u}_{n}^{h k}=\mathbf{u}_{0}^{h k}+\sum_{j=1}^{n} k \mathbf{v}_{j}^{h k}$.

In a similar way, the discrete version of the penalty Problem $\widetilde{P}_{V}^{\varepsilon}$ can be formulated as follows.

Problem $\widetilde{P}_{V \varepsilon}^{h k}$. Find a velocity field $\mathbf{v}_{\varepsilon}^{h k}=\left\{\mathbf{v}_{\varepsilon_{n}}^{h k}\right\}_{n=0}^{N} \subset V^{h}$ and a friction stress field $\boldsymbol{\lambda}_{\varepsilon}^{h k}=\left\{\boldsymbol{\lambda}_{\varepsilon_{n}}^{h k}\right\}_{n=0}^{N} \subset Y_{\tau}^{h}$ such that, for all $n=1, \ldots, N$

$$
\begin{aligned}
& \left(\frac{\rho}{k}\left(\mathbf{v}_{\varepsilon_{n}}^{h k}-\mathbf{v}_{\varepsilon_{(n-1)}}^{h k}\right), \mathbf{w}^{h}\right)_{H}+\left(\mathcal{A} \varepsilon\left(\mathbf{v}_{\varepsilon_{n}}^{h k}\right), \varepsilon\left(\mathbf{w}^{h}\right)\right)_{Q}+\left(\mathcal{B} \varepsilon\left(\mathbf{u}_{\varepsilon_{n}}^{h k}\right), \varepsilon\left(\mathbf{v}^{h}\right)\right)_{Q} \\
& \quad+\frac{1}{\varepsilon} \int_{\Gamma_{3}}\left(v_{\varepsilon_{\nu n}}^{h k}-g\right)_{+} w_{\nu}^{h} d a+\int_{\Gamma_{3}} p\left(v_{\varepsilon_{\nu n}}^{h k}\right) w_{\nu}^{h} d a \\
& \quad+\int_{\Gamma_{3}} \boldsymbol{\lambda}_{\varepsilon_{n}}^{h k} \cdot \mathbf{w}_{\tau}^{h k} d a=\left(\mathbf{f}_{n}^{h k}, \mathbf{w}^{h}\right)_{V} \quad \forall \mathbf{w}^{h} \in V^{h} \\
& \lambda_{\varepsilon_{n}}^{h k} \in \mu p\left(v_{\varepsilon_{\nu n}}^{h k}\right) \partial \varphi_{\tau}\left(\mathbf{v}_{\varepsilon_{\tau n}}^{h k}\right) \quad \text { in } \quad Y_{\tau}^{h}
\end{aligned}
$$

Here, $\mathbf{u}_{\varepsilon_{0}}^{h k}=\mathbf{u}_{0}^{h}, \mathbf{v}_{\varepsilon_{0}}^{h k}=\mathbf{u}_{1}^{h}$ and $\mathbf{u}_{\varepsilon_{n}}^{h k}=\mathbf{u}_{\varepsilon_{0}}^{h k}+\sum_{j=1}^{n} k \mathbf{v}_{\varepsilon_{j}}^{h k}$.

\subsection{The solution algorithm}

The algorithm we use to solve the discrete frictional contact Problems $\widetilde{P}_{V}^{h k}$ and $\widetilde{P}_{V \varepsilon}^{h k}$ is based on a combination of the augmented Lagrangian method for the unilateral conditions in velocity and the penalty method for the normal damped response condition. For friction law $(4.1)_{2}$, we also use an augmented Lagrangian approach, see (Alart et al., 1991; Khenous et al., 2006; Wriggers, 2002). To this end, we introduce the notation $\boldsymbol{\lambda}=\lambda_{\nu} \boldsymbol{\nu}+\boldsymbol{\lambda}_{\tau}$, where $\lambda_{\nu}=\boldsymbol{\lambda} \cdot \boldsymbol{\nu}$ and $\boldsymbol{\lambda}_{\tau}=\boldsymbol{\lambda}-\lambda_{\nu} \boldsymbol{\nu}$. We now introduce the expressions of the functions $\mathbf{w}^{h}, \mathbf{u}^{h}$ and $\boldsymbol{\delta} \mathbf{u}^{h}$ by considering 
their values at the $i$-th node of $\mathcal{T}^{h}$ and the basis functions $\alpha^{i}$ of the space $V^{h}$ for $i=1, \ldots, N_{\text {tot }}^{h}$, i.e.

$$
\mathbf{w}^{h}=\sum_{i=1}^{N_{\text {tot }}^{h}} \mathbf{w}^{i} \alpha^{i} \quad \mathbf{u}^{h}=\sum_{i=1}^{N_{\text {tot }}^{h}} \mathbf{u}^{i} \alpha^{i} \quad \boldsymbol{\delta} \mathbf{u}^{h}=\sum_{i=1}^{N_{\text {tot }}^{h}} \boldsymbol{\delta} \mathbf{u}^{i} \alpha^{i}
$$

Here and below, $N_{\text {tot }}^{h}$ represents the total number of nodes of $\mathcal{T}^{h}$, and $N_{\Gamma_{3}}^{h}$ denotes the total number of nodes of $\mathcal{T}^{h}$ lying on $\Gamma_{3}$.

The augmented Lagrangian approach shows us that the Problem $\widetilde{P}_{V}^{h k}$ can be governed by the system of nonlinear equations

$$
\mathbf{R}\left(\delta \mathbf{v}_{n}, \mathbf{v}_{n}, \mathbf{u}_{n}, \boldsymbol{\lambda}_{n}\right)=\widetilde{\mathbf{M}}\left(\delta \mathbf{v}_{n}\right)+\widetilde{\mathbf{A}}\left(\mathbf{v}_{n}\right)+\widetilde{\mathbf{G}}\left(\mathbf{u}_{n}\right)+\mathcal{F}\left(\mathbf{v}_{n}, \boldsymbol{\lambda}_{n}\right)=\mathbf{0}
$$

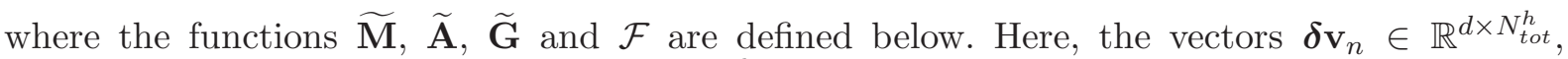
$\mathbf{v}_{n} \in \mathbb{R}^{d \times N_{\text {tot }}^{h}}, \mathbf{u}_{n} \in \mathbb{R}^{d \times N_{\text {tot }}^{h}}$ and $\boldsymbol{\lambda}_{n} \in \mathbb{R}^{d \times N_{\Gamma_{3}}^{h}}$ represent the generalized velocity, the displacement and the Lagrange multiplier vectors defined by

$$
\begin{array}{lll}
\boldsymbol{\delta} \mathbf{v}_{n}=\left\{\boldsymbol{\delta} \mathbf{v}_{n}^{i}\right\}_{i=1}^{N_{t o t}^{h}} & \mathbf{v}_{n}=\left\{\mathbf{v}_{n}^{i}\right\}_{i=1}^{N_{\text {tot }}^{h}} \\
\boldsymbol{\lambda}_{n}=\left\{\boldsymbol{\lambda}_{n}^{i}\right\}_{i=1}^{N_{\Gamma_{3}}^{h}} \quad \text { for all } \quad n=1, \ldots, N & \mathbf{u}_{n}=\left\{\mathbf{u}_{n}^{i}\right\}_{i=1}^{N_{\text {tot }}^{h}}
\end{array}
$$

where $\delta \mathbf{v}_{n}^{i}, \mathbf{v}_{n}^{i}$ and $\mathbf{u}_{n}^{i}$ denote values of the functions $\delta \mathbf{v}_{n}^{h k}, \mathbf{v}_{n}^{h k}$ and $\mathbf{u}_{n}^{h k}$ at the $i$-th nodes of $\mathcal{T}^{h}$. Moreover, $\boldsymbol{\lambda}_{n}^{i}$ represents the value of $\boldsymbol{\lambda}_{n}^{h k}$ at the $i$-th node of the discretized contact interface. Next, the generalized acceleration term $\widetilde{\mathbf{M}}(\mathbf{a}) \in \mathbb{R}^{d \times N_{\text {tot }}^{h}} \times \mathbb{R}^{d \times N_{\Gamma_{3}}^{h}}$, the generalized viscous term $\widetilde{\mathbf{A}}(\mathbf{v}) \in \mathbb{R}^{d \times N_{\text {tot }}^{h}} \times \mathbb{R}^{d \times N_{\Gamma_{3}}^{h}}$ and the generalized elastic term $\widetilde{\mathbf{G}}(\mathbf{u}) \in \mathbb{R}^{d \times N_{\text {tot }}^{h}} \times \mathbb{R}^{d \times N_{\Gamma_{3}}^{h}}$ are defined by $\widetilde{\mathbf{M}}(\mathbf{a})=\left(\mathbf{M}(\mathbf{a}), \mathbf{0}_{d \times N_{\Gamma_{3}}^{h}}\right), \widetilde{\mathbf{A}}(\mathbf{v})=\left(\mathbf{A}(\mathbf{v}), \mathbf{0}_{d \times N_{\Gamma_{3}}^{h}}\right)$ and $\widetilde{\mathbf{G}}(\mathbf{u})=\left(\mathbf{G}(\mathbf{u}), \mathbf{0}_{d \times N_{\Gamma_{3}}^{h}}\right)$. Here $\mathbf{0}_{d \times N_{\Gamma_{3}}^{h}}$ is the zero element of $\mathbb{R}^{d \times N_{\Gamma_{3}}^{h}}$; also, $\mathbf{M}(\mathbf{a}) \in \mathbb{R}^{d \times N_{\text {tot }}^{h}}, \mathbf{A}(\mathbf{v}) \in \mathbb{R}^{d \times N_{\text {tot }}^{h}}$ and $\mathbf{G}(\mathbf{u}) \in \mathbb{R}^{d \times N_{\text {tot }}^{h}}$ denote the acceleration term, the viscous term and the elastic term, respectively, given by

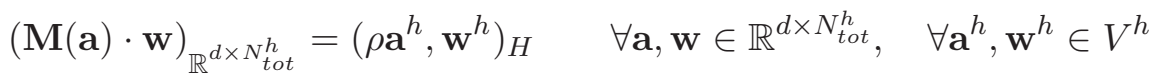

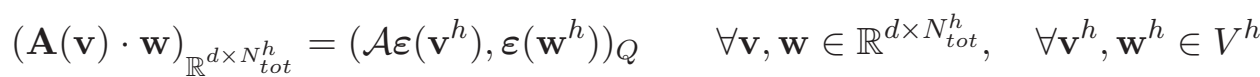

$$
\begin{aligned}
& (\mathbf{G}(\mathbf{u}) \cdot \mathbf{w})_{\mathbb{R}^{d \times N_{\text {tot }}^{h}}}=\left(\mathcal{B} \varepsilon\left(\mathbf{u}^{h}\right), \varepsilon\left(\mathbf{w}^{h}\right)\right)_{Q}-\left(\mathbf{f}_{n}, \mathbf{w}^{h}\right)_{V} \quad \forall \mathbf{u}, \mathbf{w} \in \mathbb{R}^{d \times N_{t o t}^{h}}, \quad \forall \mathbf{u}^{h}, \mathbf{w}^{h} \in V^{h}
\end{aligned}
$$

Above, $\mathbf{a}, \mathbf{v}, \mathbf{u}$ and $\mathbf{w}$ represent the generalized vectors of the components $\mathbf{a}_{i}, \mathbf{v}_{i}, \mathbf{u}_{i}$ and $\mathbf{w}_{i}$, for $i=1, \ldots, N_{t o t}^{h}$, respectively, and note that the volume and surface efforts are contained in the term $\mathbf{G}\left(\mathbf{u}_{n}\right)$. Finally, the frictional contact operator $\mathcal{F}\left(\mathbf{v}_{n}, \boldsymbol{\lambda}_{n}\right)$ associated to the boundary condition on the contact surface is given by

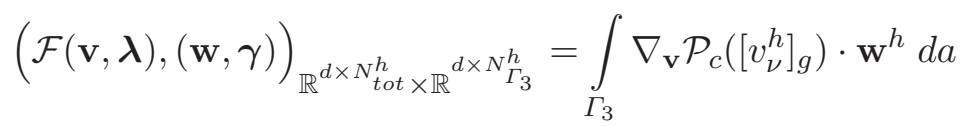

$$
\begin{aligned}
& +\int_{\Gamma_{3}} \nabla_{\mathbf{v}}\left(l_{\nu}^{r}\left(\mathbf{v}^{h}, \boldsymbol{\lambda}^{h}\right)+l_{\tau}^{r}\left(\mathbf{v}^{h}, \boldsymbol{\lambda}^{h}\right)\right) \cdot \mathbf{w}^{h} d a+\int_{\Gamma_{3}} \nabla_{\boldsymbol{\lambda}}\left(l_{\nu}^{r}\left(\mathbf{v}^{h}, \boldsymbol{\lambda}^{h}\right)+l_{\tau}^{r}\left(\mathbf{v}^{h}, \boldsymbol{\lambda}^{h}\right)\right) \cdot \gamma^{h} d a \\
& \forall \mathbf{v}, \mathbf{w} \in \mathbb{R}^{d \times N_{t o t}^{h}}, \quad \forall \boldsymbol{\lambda}, \boldsymbol{\gamma} \in \mathbb{R}^{d \times N_{\Gamma_{3}}^{h}}, \quad \forall \mathbf{v}^{h}, \mathbf{w}^{h} \in V^{h}, \quad \forall \boldsymbol{\lambda}^{h}, \gamma^{h} \in Y_{\nu}^{h} \times Y_{\tau}^{h}
\end{aligned}
$$

Here $\mathcal{P}_{c}: \mathbb{R} \rightarrow \mathbb{R}$ is a derivable function such that $\nabla_{\mathbf{v}} \mathcal{P}_{c}=p$ on $(-\infty, g],[\cdot]_{g}: \mathbb{R} \rightarrow \mathbb{R}$ is the function defined by

$$
[s]_{g}=\left\{\begin{array}{lll}
s & \text { if } & s \leqslant g \\
g & \text { if } & s>g
\end{array}\right.
$$


and $\nabla_{\mathbf{x}}$ represents the gradient operator with respect the variable $\mathbf{x}$. Also $l_{\nu}^{r}$ and $l_{\tau}^{r}$ denote the augmented Lagrangian functionals

$$
\begin{aligned}
& l_{\nu}^{r}\left(\mathbf{v}^{h}, \lambda_{\nu}^{h}\right)=v_{\nu}^{h} \lambda_{\nu}^{h}+\frac{r_{\nu}}{2}\left(v_{\nu}^{h}-g\right)^{2}-\frac{1}{2 r_{\nu}} \operatorname{dist}^{2}\left\{\lambda_{\nu}+r_{\nu}\left(v_{\nu}^{h}-g\right), \mathbb{R}_{-}^{d}\right\}, \\
& l_{\tau}^{r}\left(\mathbf{v}^{h}, \boldsymbol{\lambda}_{\tau}^{h}\right)=\mathbf{v}_{\tau}^{h} \cdot \boldsymbol{\lambda}_{\tau}^{h}+\frac{r_{\tau}}{2}\left|\mathbf{v}_{\tau}^{h}\right|^{2}-\frac{1}{2 r_{\tau}} \operatorname{dist}^{2}\left\{\boldsymbol{\lambda}_{\tau}^{h}+r_{\tau} \mathbf{v}_{\tau}^{h}, C\left[\mu p\left(v_{\nu}^{h}\right)\right]\right\}
\end{aligned}
$$

Here, $r_{\nu}$ and $r_{\tau}$ are positive penalty coefficients, $C\left[\mu p\left(v_{\nu}^{h}\right)\right]$ represents the convex disk of constant radius $\mu p\left(v_{\nu}^{h}\right)$ and $\operatorname{dist}\{x, C\}$ denotes the distance from $x$ to the set $C$, i.e., $\operatorname{dist}\{x, C\}=\inf _{y \in C}\|x-y\|$.

Note that, in the case of penalty contact condition (2.28), there is no need to use the Lagrange method. Indeed, the penalty method can be used by considering $\lambda_{\nu}=0$ in equation $(4.8)_{1}$. Then, augmented Lagrangian functional (4.8) 1 takes a simpler expression

$$
l_{\nu}^{r}\left(\mathbf{v}^{h}, 0\right)=\frac{r_{\nu}}{2}\left(v_{\nu}^{h}-g\right)^{2}-\frac{1}{2 r_{\nu}} \operatorname{dist}^{2}\left\{r_{\nu}\left(v_{\nu}^{h}-g\right), \mathbb{R}_{-}^{d}\right\}
$$

in which $r_{\nu}$ can be replaced by the penalty parameter $\varepsilon$. Thus, the frictional contact operator $\mathcal{F}(\mathbf{v}, \boldsymbol{\lambda})$ associated with frictional contact conditions (2.28) and (2.29) is given by

$$
\begin{aligned}
& (\mathcal{F}(\mathbf{v}, \boldsymbol{\lambda}),(\mathbf{w}, \gamma))_{\mathbb{R}^{d \times N_{\text {tot } \times \mathbb{R}}^{h}}{ }^{d \times N_{\Gamma_{3}}^{h}}}=\int_{\Gamma_{3}} \nabla_{\mathbf{v}} \mathcal{P}_{c}\left(\left[v_{\nu}^{h}\right]_{g}\right) \cdot \mathbf{w}^{h} d a \\
& +\frac{1}{\varepsilon} \int_{\Gamma_{3}}\left(v_{\nu}^{h}-g\right)_{+} w_{\nu}^{h} d a+\int_{\Gamma_{3}} \nabla_{\mathbf{v}} l_{\tau}^{r}\left(\mathbf{v}^{h}, \boldsymbol{\lambda}^{h}\right) \cdot \mathbf{w}^{h} d a+\int_{\Gamma_{3}} \nabla_{\boldsymbol{\lambda}} l_{\tau}^{r}\left(\mathbf{v}^{h}, \boldsymbol{\lambda}^{h}\right) \cdot \gamma^{h} d a \\
& \forall \mathbf{v}, \mathbf{w} \in \mathbb{R}^{d \times N_{\text {tot }}^{h}}, \quad \forall \boldsymbol{\lambda}, \boldsymbol{\gamma} \in \mathbb{R}^{d \times N_{\Gamma_{3}}^{h}}, \quad \forall \mathbf{v}^{h}, \mathbf{w}^{h} \in V^{h}, \quad \forall \boldsymbol{\lambda}^{h}, \gamma^{h} \in Y_{\nu}^{h} \times Y_{\tau}^{h}
\end{aligned}
$$

The solution algorithm consists in a prediction-correction scheme based on a finite differences method (the backward Euler difference method) and a linear iterations method (the Newton method). The finite difference scheme we use is characterized by a first order time integration scheme, both for the acceleration $\delta \mathbf{v}_{n}$ and the velocity $\mathbf{v}_{n}=\delta \mathbf{u}_{n}$. To solve nonlinear system (4.7), at each time increment the variables $\left(\mathbf{v}_{n}, \boldsymbol{\lambda}_{n}\right)$ are treated simultaneously through the Newton method. For this reason, we use in what follows the notation $\mathbf{x}_{n}=\left(\mathbf{v}_{n}, \boldsymbol{\lambda}_{n}\right)$. Inside the loop of the increment of time indexed by $n$, the algorithm can be developed in three steps which are the following.

For $n=0$ until $N$, let $\mathbf{u}_{0}, \mathbf{v}_{0}$ and $\boldsymbol{\lambda}_{0}$ be given.

- The prediction step: This step provides the initial values $\mathbf{u}_{n+1}^{0}, \mathbf{v}_{n+1}^{0}$ and $\boldsymbol{\lambda}_{n+1}^{0}$ by the formulas

$$
\mathbf{u}_{n+1}^{0}=\mathbf{u}_{n} \quad \boldsymbol{\lambda}_{n+1}^{0}=\boldsymbol{\lambda}_{n} \quad \mathbf{v}_{n+1}^{0}=\mathbf{0}
$$

- The Newton linearization step: At the iteration $i$ of the Newton method, we have

$$
\mathbf{x}_{n+1}^{i+1}=\mathbf{x}_{n+1}^{i}-\left(\frac{\mathbf{C}_{n+1}^{i}}{k}+\mathbf{Q}_{n+1}^{i}+k \mathbf{K}_{n+1}^{i}+\mathbf{T}_{n+1}^{i}\right)^{-1} \mathbf{R}\left(\frac{\mathbf{v}_{n+1}^{i}-\mathbf{v}_{n}^{i}}{k}, \mathbf{v}_{n+1}^{i}, \mathbf{u}_{n+1}^{i}, \lambda_{n+1}^{i}\right)
$$

where $\mathbf{x}_{n+1}^{i+1}$ denotes the pair $\left(\mathbf{v}_{n+1}^{i+1}, \boldsymbol{\lambda}_{n+1}^{i+1}\right) ; i$ and $n$ represent respectively the Newton iteration index and the time index, respectively; $\mathbf{C}_{n+1}^{i}=D_{\mathbf{v}} \mathbf{M}\left(\delta \mathbf{v}_{n+1}^{i}\right)$ denotes the mass matrix, $\mathbf{Q}_{n+1}^{i}=D_{\mathbf{v}} \mathbf{A}\left(\mathbf{v}_{n+1}^{i}\right)$ is the damping matrix, $\mathbf{K}_{n+1}^{i}=D_{\mathbf{v}} \mathbf{G}\left(\mathbf{u}_{n+1}^{i}\right)$ represents the elastic matrix and $\mathbf{T}_{n+1}^{i}=D_{\mathbf{v}, \lambda} \mathcal{F}\left(\mathbf{v}_{n+1}^{i}, \boldsymbol{\lambda}_{n+1}^{i}\right)$ is the contact tangent matrix. Also, $D_{\mathbf{v}} \mathbf{M}, D_{\mathbf{v}} \mathbf{A}, D_{\mathbf{v}} \mathbf{G}$ and 
$D_{\mathbf{v}, \lambda} \mathcal{F}$ denote the differentials of the functions $\mathbf{M}, \mathbf{A}, \mathbf{G}$ and $\mathcal{F}$ according to the variables $\mathbf{v}$ and $\boldsymbol{\lambda}$. This leads us to solve the resulting linear system

$$
\left(\frac{\mathbf{C}_{n+1}^{i}}{k}+\mathbf{Q}_{n+1}^{i}+k \mathbf{K}_{n+1}^{i}+\mathbf{T}_{n+1}^{i}\right) \Delta \mathbf{x}^{i}=-\mathbf{R}\left(\frac{\mathbf{v}_{n+1}^{i}-\mathbf{v}_{n}^{i}}{k}, \mathbf{v}_{n+1}^{i}, \mathbf{u}_{n+1}^{i}, \boldsymbol{\lambda}_{n+1}^{i}\right)
$$

where $\Delta \mathbf{x}=\left(\Delta \mathbf{v}^{i}, \Delta \boldsymbol{\lambda}^{i}\right)$ with $\Delta \mathbf{v}^{i}=\mathbf{v}_{n+1}^{i+1}-\mathbf{v}_{n+1}^{i}$ and $\Delta \boldsymbol{\lambda}^{i}=\boldsymbol{\lambda}_{n+1}^{i+1}-\boldsymbol{\lambda}_{n+1}^{i}$.

- The correction step: Once system (4.10) is solved, we update $\mathbf{x}_{n+1}^{i+1}$ and $\mathbf{u}_{n+1}^{i+1}$ by

$$
\mathbf{x}_{n+1}^{i+1}=\mathbf{x}_{n+1}^{i}+\Delta \mathbf{x}^{i} \quad \mathbf{u}_{n+1}^{i+1}=\mathbf{u}_{n+1}^{i}+k \Delta \mathbf{v}^{i}
$$

Note that formulation (4.7) has been implemented in the open-source finite element library GetFEM++ (see http://getfem.org/).

\section{Numerical simulations}

To verify the performance and the accuracy of the numerical method described in the previous Section, a number of numerical experiments have been performed on a well known test problem. We describe in this Section the numerical results we obtained for Problems $P_{V}$ and $P_{V \varepsilon}$ in dimension two. The physical setting is depicted in Fig. 1. There, the domain $\Omega=(0,2) \times(0,1)$

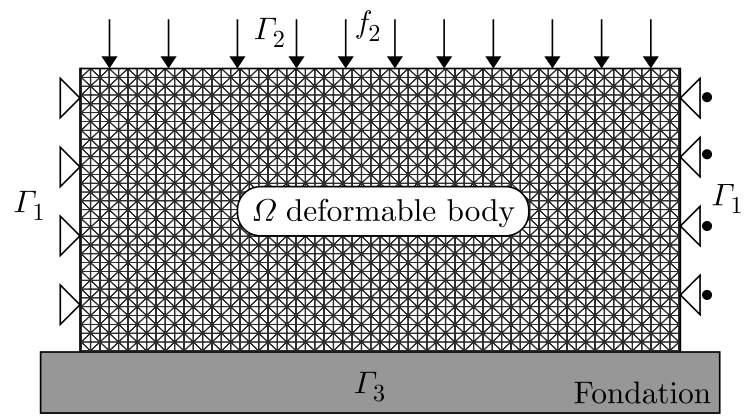

Fig. 1. Physical setting and finite element discretization for $h=1 / 33$

is the cross section of a three-dimensional linearly viscoelastic body subjected to the action of tractions in such a way that a plane stress hypothesis is assumed. On the part $\{0\} \times[0,1]$, the body is clamped and, therefore, the displacement field vanishes there; the horizontal component of the displacement field vanishes on the part $\{2\} \times[0,1]$. Thus, $\Gamma_{1}=(\{0\} \times[0,1]) \cup(\{2\} \times[0,1])$. Vertical tractions act on the part $\Gamma_{2}$ of the boundary. No body forces are assumed to act on the viscoelastic body during the dynamic process. The body is in frictional contact with a rigid obstacle on the part $\Gamma_{3}=[0,2] \times\{0\}$ of the boundary. We recall that the contact follows the normal damped response condition associated to Coulomb's law of dry friction as far as the normal velocity is less than the bound $g$ and, when this bound is reached, it follows a unilateral condition in velocity associated to the Tresca friction law.

The material response is governed by a viscoelastic linear constitutive law defined by the elasticity tensor $\mathcal{B}$ and the viscosity tensor $\mathcal{A}$ given by

$$
\begin{array}{ll}
(\mathcal{B} \tau)_{\alpha \beta}=\frac{E \nu}{1-\kappa^{2}}\left(\tau_{11}+\tau_{22}\right) \delta_{\alpha \beta}+\frac{E}{1+\kappa} \tau_{\alpha \beta} & \\
(\mathcal{A} \tau)_{\alpha \beta}=\mu\left(\tau_{11}+\tau_{22}\right) \delta_{\alpha \beta}+\eta \tau_{\alpha \beta} \quad 1 \leqslant \alpha & \beta \leqslant 2
\end{array}
$$

where $E$ is Young's modulus, $\kappa$ is Poisson's ratio of the material, $\mu, \eta$ are viscosity constants, and $\delta_{\alpha \beta}$ denotes the Kronecker symbol. 
In computations, we have used the following data: $T=1 \mathrm{~s}, \mathbf{u}_{0}=\mathbf{0} \mathrm{m}, \dot{\mathbf{u}}_{0}=\mathbf{0} \mathrm{m} / \mathrm{s}$, $\rho=2000 \mathrm{Kg} / \mathrm{m}^{3}, E=100 \mathrm{GPa}, \nu=0.2, \mu_{1}=0.25 \mathrm{GPa}, \mu_{2}=0.5 \mathrm{GPa}, \mathbf{f}_{0}=(0,0) \mathrm{GPa}$, $\mathbf{f}_{2}=(0,-10 t) \mathrm{GPam}$ on $\Gamma_{2}, \mu=0.4, g=0.05 \mathrm{~m} / \mathrm{s}, p(r)=c_{\nu}(r)_{+}, c_{\nu}=50 \mathrm{GPas}$, $\varepsilon=1 / 50000$ GPas.
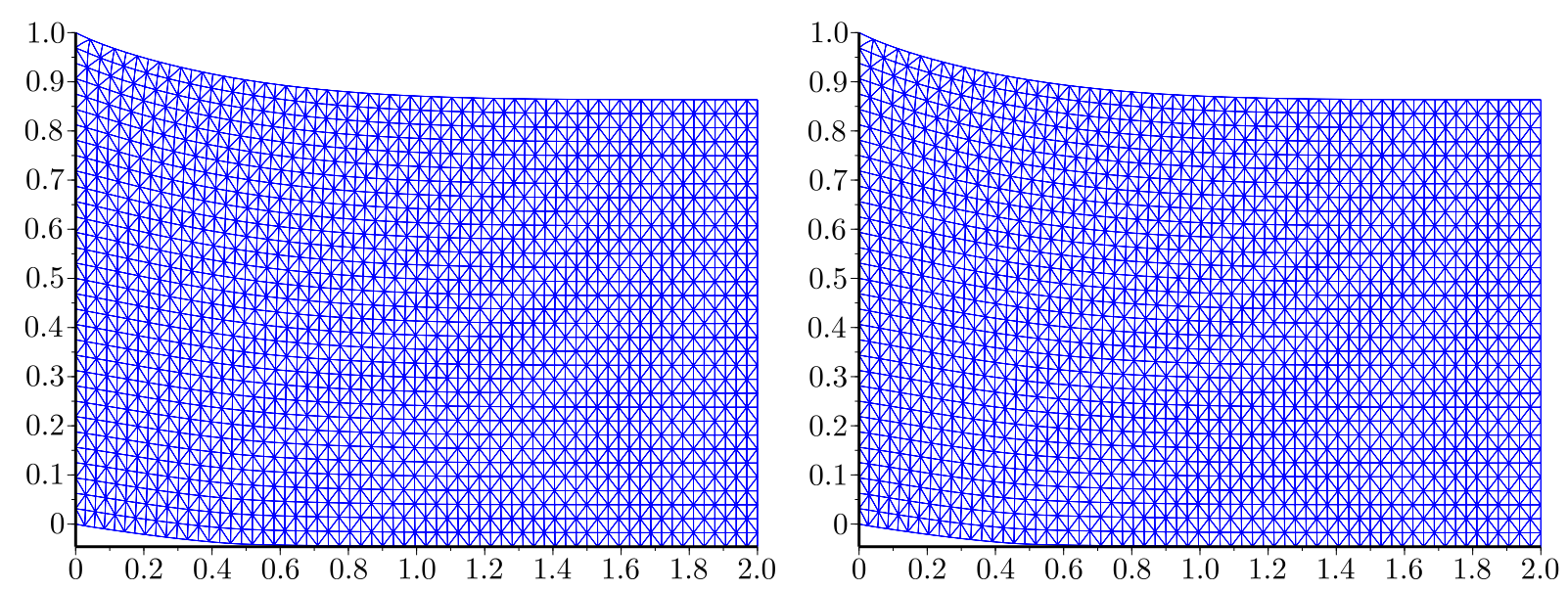

Fig. 2. Deformed configuration at the final time: the original contact problem (left) and the penalty problem (right)

The deformed configuration of the body at the final time $T=1 \mathrm{~s}$ is represented in Fig. 2 (left), which corresponds to the numerical solution of problem $P_{V}$. Note that the vertical displacement of the bottom side is quite large, and this is a consequence of the fact that we model the contact with the normal damped response condition which, in contrast to the unilateral condition in displacement, describes the contact with a deformable foundation and allows penetration. In order to compare the deformed mesh related to Problem $P_{V}$ with that obtained for the numerical solution of Problem $P_{V}^{\varepsilon}$, we plotted in Fig. 2 (right) the deformed configuration for the numerical solution of the penalty problem $P_{V}^{\varepsilon}$. Then, in Figs. 3 and 4 , we show the reactions and velocities of the nodes of the contact surface for $\mu=0.4$. The zone AB is a sliding zone formed by 15 nodes which are in a status of the normal damped response; there, the normal velocity is such that $0<\dot{u}_{\nu}<g$ and the tangential velocity does not vanishes, i.e., $\dot{\mathbf{u}}_{\tau} \neq \mathbf{0}$. In this zone, the friction follows the Coulomb law. The zone BC is a sliding zone formed of 29 nodes which are in a status of the unilateral condition in velocity; there, the normal velocity reaches the bound $g$. In this zone, the friction follows the Tresca law with the friction bound $F_{b}$. Next, we have the stick zone $\mathrm{CD}$ where the slip vanishes and $\left\|\boldsymbol{\sigma}_{\tau}\right\|<F_{b}$.
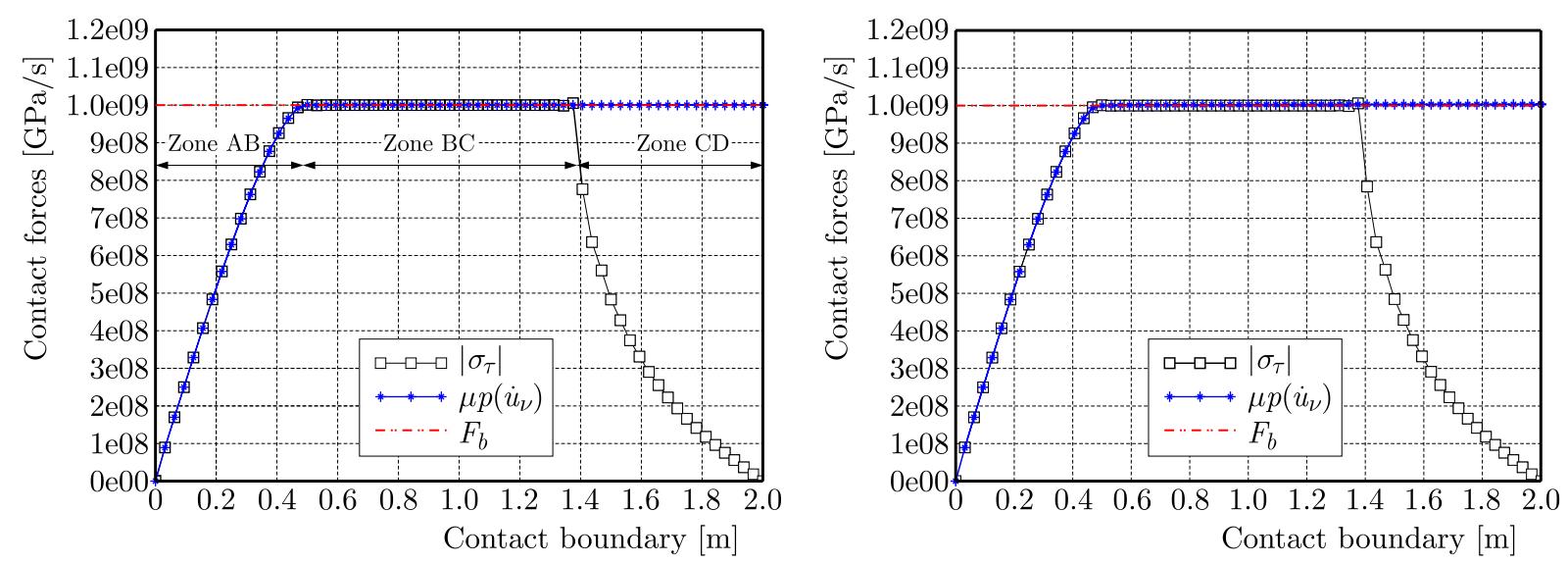

Fig. 3. Frictional contact reactions on $\Gamma_{3}$ at the final time: the original contact problem (left) and the penalty problem (right) 

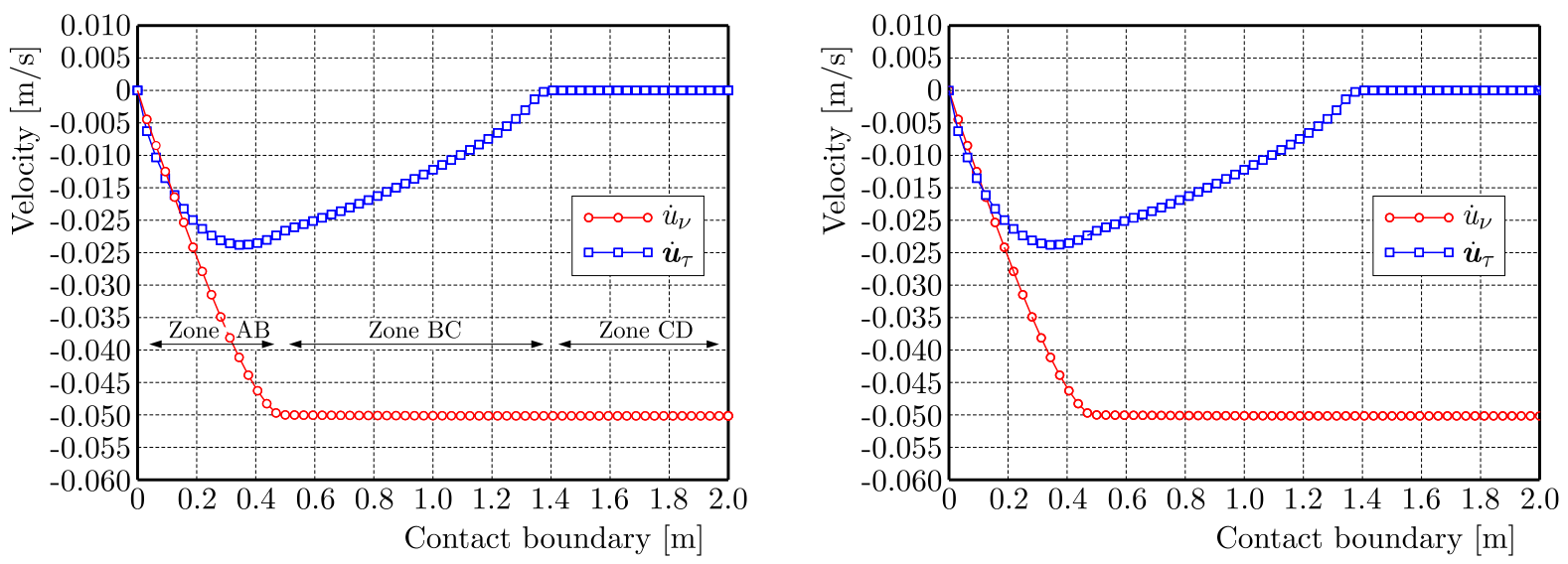

Fig. 4. Velocity on $\Gamma_{3}$ at final time: the original contact problem (left) and the penalty problem (right)

According to the deformed configurations (Figs. 2-4), we observe that the numerical results obtained for the solution of Problem $P_{V}$ are very well approximated by the solution of Problem $P_{V \epsilon}$. Next, we lead a parametric study according to the penalty coefficient $\varepsilon$. To this end, in Fig. 5 we consider various values of $\varepsilon$, and we compare in the left graph the normal velocities profiles on the contact boundary obtained for the solution of the original contact Problem $P_{V}$ and the penalty Problem $P_{V}^{\varepsilon}$. In the right graph of Fig. 5, we study the convergence on the whole discrete domain $\Omega^{h}$ of the velocity solution obtained for Problem $P_{V}^{\varepsilon}$ towards that obtained for Problem $P_{V}$. Here, we consider the numerical estimation of the difference $\left\|\dot{\mathbf{u}}_{\varepsilon}^{h k}-\dot{\mathbf{u}}^{h k}\right\|$ at the time $T=1 \mathrm{~s}$ between the numerical solutions obtained for Problems $P_{V}$ and $P_{V}^{\varepsilon}$. The results depicted in Fig. 5 illustrate that the solution of the penalty problem gives a reliable and accurate approximation of the original problem, provided that the penalty parameter takes very large values.
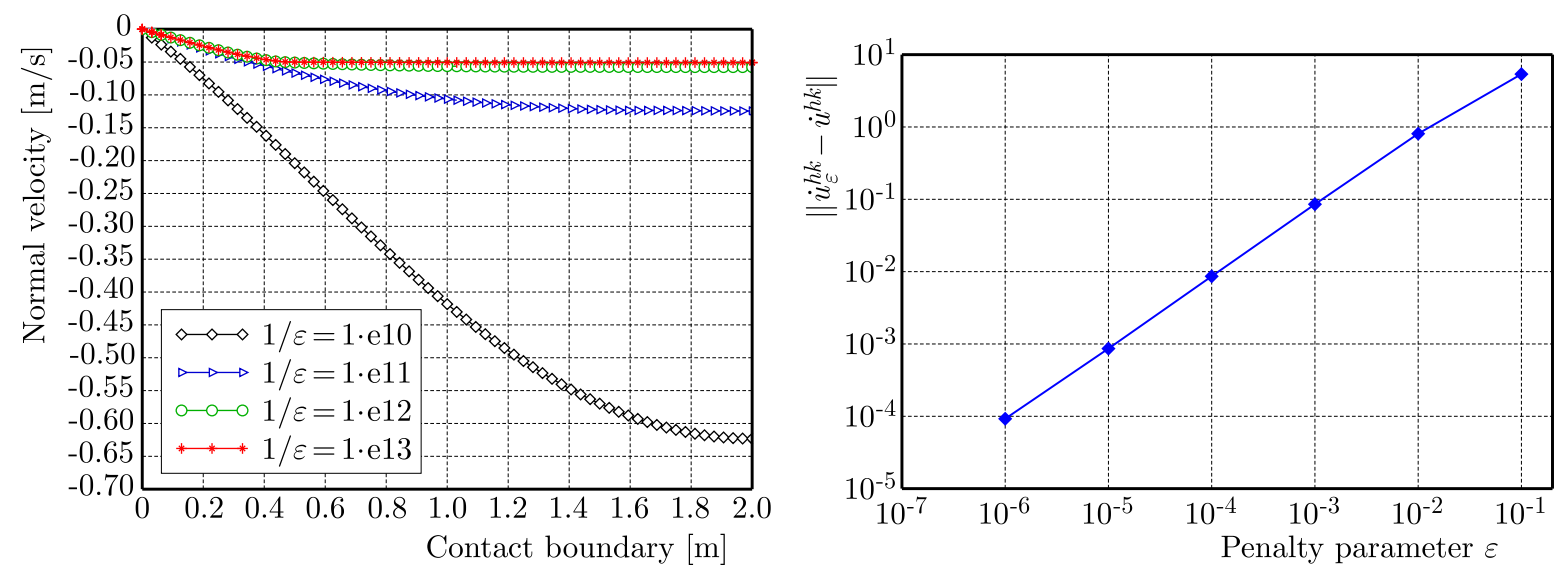

Fig. 5. Normal velocity for different values of the contact penalty parameter (left) and convergence of the penalty method (right)

\section{References}

1. Alart P., Curnier A., 1991, A mixed formulation for frictional contact problems prone to Newton like solution methods, Computer Methods in Applied Mechanics and Engineering, 92, 3, 353-375

2. Barboteu M., Bartosz K., Kalita P., 2015, A dynamic viscoelastic contact problem with normal compliance, finite penetration and nonmonotone slip rate dependent friction, Nonlinear Analysis: Real World Applications, 22, 452-472 
3. Barboteu M., Cheng X.L., Sofonea M., 2016a, Analysis of a contact problem with unilateral constraint and slip-dependent friction, Mathematics and Mechanics of Solids, 21, 791-811

4. Barboteu M., Danan D., 2016, Analysis of a dynamic viscoelastic contact problem with normal compliance, normal damped response, and nonmonotone slip rate dependent friction, Advances in Mathematical Physics, 2016, http://dx.doi.org/10.1155/2016/1562509

5. Barboteu M., Danan D., Sofonea M., 2016b, Analysis of a contact problem with normal damped response and unilateral constraint, Zeitschrift für Angewandte Mathematik and Mechanik, 96, 408-428

6. Chouly F., Hild P., 2013, On convergence of the penalty method for unilateral contact problems, Applied Numerical Mathematics, 65, 27-40

7. Duvaut G., Lions J.L., 1976, Inequalities in Mechanics and Physics, Springer Verlag, Berlin

8. HAN J., Migórski S., Zeng H., 2016, Analysis of a dynamic viscoelastic unilateral contact problem with normal damped response, Nonlinear Analysis: Real World Applications, 28, 229-250

9. Han W., Shillor M., Sofonea M., 2001, Variational and numerical analysis of a quasistatic viscoelastic problem with normal compliance, friction and damage, Journal of Computational and Applied Mathematics, 137, 377-398

10. Han W., Sofonea M., 2002, Quasistatic Contact Problems in Viscoelasticity and Viscoplasticity, American Mathematical Society-International Press

11. HlaváČé I., Haslinger J., NeČas J., Lovíšek J., 1988, Solution of Variational Inequalities in Mechanics, Springer, New York, NY, USA.

12. Khenous H.B., Laborde P., Renard Y., 2006, On the discretization of contact problems in elastodynamics, [In:] Analysis and Simulation of Contact Problems, 27, 31-38, Lecture Notes in Applied and Computational Mechanics, Springer, Berlin, Germany

13. Kikuchi N., Song Y., 1981, Penalty finite element approximations of a class of unilateral problems in linear elasticity, Quarterly of Applied Mechanics, 39, 1-21

14. Laursen T.A., 2002, Computational Contact and Impact Mechanics, Springer, Berline, Germany

15. Oden J.T., Martins J.A.C., 1985, Models and computational methods for dynamic friction phenomena, Computer Methods in Applied Mechanics and Engineering, 52, 527-634

16. Shillor M., Sofonea M., Telega J., 2004, Models and Variational Analysis of Quasistatic Contact, 655, Lecture Notes in Physics, Springer, Berlin, Germany

17. Sofonea M., Matei A., 2012, Mathematical Models in Contact Mechanics, 398, London Mathematical Society Lecture Note Series, Cambridge University Press, Cambridge

18. Wriggers P., 2002, Computational Contact Mechanics, John Wiley \& Sons, Chichester, UK 